\title{
Variational Reduction for the Transport Equation in a Multiple Branching Plants Growth Model
}

\author{
S. Boujena ${ }^{1 *}$, A. Chiboub ${ }^{1}$ and J. Pousin ${ }^{2}$ \\ ${ }^{1}$ Department of Mathematics and Informatique, Faculty of Sciences Ain Chock, \\ B.P 5366, Maarif 20200, Casablanca-Morocco \\ 2 INSA of Lyon, Institute Camille Jordan, UMR 5208, 69100 Villeurbanne, France
}

\begin{abstract}
Plant growth depends essentially on nutrients coming from the roots and metabolites produced by the plant. Appearance of new branches is determined by concentrations of certain plant hormones. The most important of them are Auxin and Cytokinin. Auxin is produced in the growing, Cytokinin in either roots or in growing parts. Many dynamical models of this phenomena have been studied in [1]. In [5], the authors deal with one branch model. In this work, we focus our interest on a multiple branch model. We deal with the transport equation in domains of different sizes. A variational reduction type method [3] based on asymptotic partial decomposition introduced in [2] (see also [4]) is used. In this work we consider the transport equation in decomposed domain with a general right hand side.
\end{abstract}

Key words: plant growth, transport equation, multiple branching plants, variational reduction, cells proliferation, concentration, hormone, displacement

AMS subject classification: 60J80, 92C80, 91B62

\section{Introduction}

The growing part of the plant contains a narrow exterior part, considered as a surface, where cells proliferate. The displacement of this surface corresponds to the plant growth. The appearance of new cells implies that old cells exit the external layer after some time and become a part of the internal plant tissue. They cannot divide any more, and they serve to conduct nutrients to the

${ }^{*}$ Corresponding author. E-mail: boujena@yahoo.fr 
meristem. The cell proliferation rate is determined by concentration of nutrients coming from the roots and metabolites produced by the plant and distributed through it. Shoots appear if the concentrations of the hormones, Auxin and Cytokinin are in a certain proportion. We consider in this paper the one-dimensional case justified if the length (or height) L of the plant is essentially greater than the diameter of its trunk. Hence we consider the interval $0 \leq x \leq L(t)$ with the length depending on time. The left endpoint $x=0$ corresponds to the root. The right endpoint, $x=L(t)$ corresponds to the apex. Its width is much less than that of the plant. It is considered in the model as a mathematical point. The interval $0<x<L(t)$ corresponds to differentiated cells that conduct nutrients from the root to the apex. We suppose that they are in liquid solution. The evolution of the concentrations of nutrients and hormones is described by a diffusion-advection equation [1].

\section{Presentation}

Our interest in this work is on a multiple branch model. We deal with the transport equation in domains of different sizes (see figure 1). A variational reduction type method [3] based on asymptotic partial decomposition is used. We consider the transport equation in heterogeneous domain with a general right hand side. A weak formulation is given on each part of this composed domain. After that we introduce the decomposed problem on the whole domain where a weak formulation is improved for the matching conditions on the interface. Introducing a variational reduction type method, we show that the decomposed problem can be reduced to a $2 D-1 D$ problem.

\subsection{Governing Equations}

Let us consider the transport equation in the domain $\mathrm{Q}$

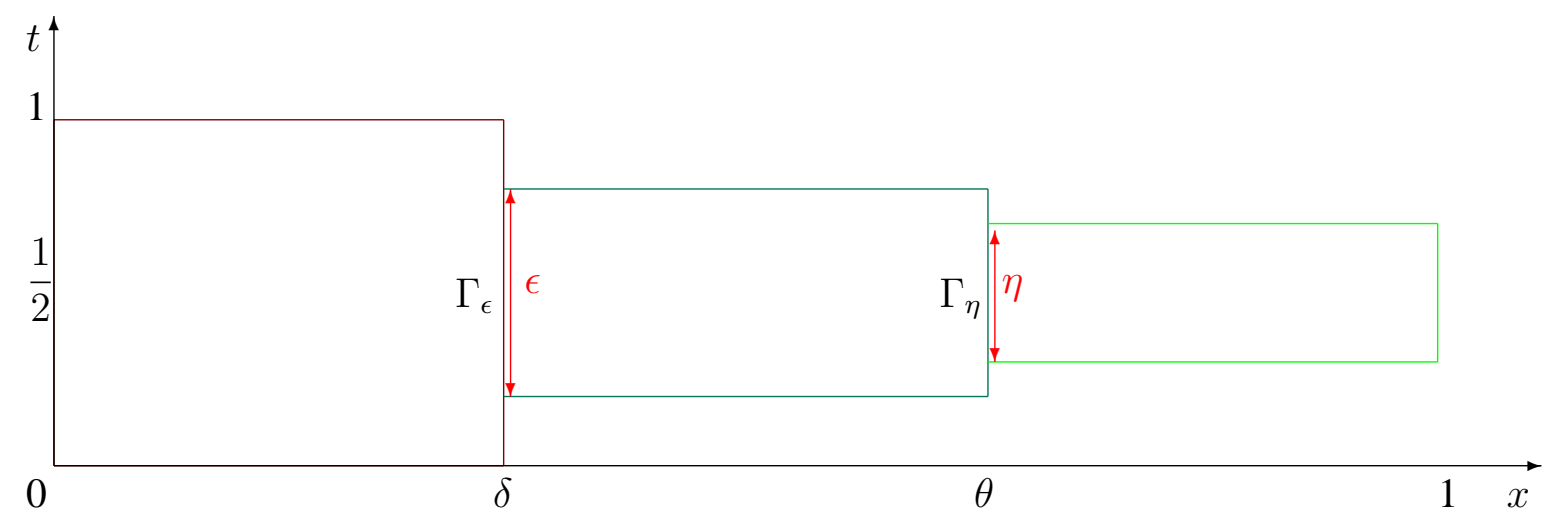

Figure 1: Decomposed domain Q

$$
\begin{aligned}
Q & =(0,1) \times(0, \delta) \cup\left(\frac{1}{2}-\epsilon, \frac{1}{2}+\epsilon\right) \times(\delta, \theta) \cup\left(\frac{1}{2}-\eta, \frac{1}{2}+\eta\right) \times(\theta, 1), \\
& =Q_{1} \cup Q_{2} \cup Q_{3} .
\end{aligned}
$$


Let $f \in C^{0}(Q, \mathbb{R})$ and $a \in C^{1}(Q ; \mathbb{R})$ be given, we define the function $\beta=\left(\begin{array}{c}1 \\ a(t, x)\end{array}\right)$ and we assume $a$ to be bounded from below by a positive number. Defining $\partial Q_{-}=\{(t, x),(\beta / n)<0\}$, we look for

$$
\begin{aligned}
u \in H(\beta, Q)= & \left\{\rho \in L^{2}(Q), \operatorname{div}(\beta \rho) \in L^{2}(Q), \rho \backslash \partial Q_{-} \in L^{2}\left(\partial Q_{-},|(\beta / n)| d \sigma\right)\right\}, \\
& \begin{cases}(\beta / \nabla u)_{2}=\frac{\partial u}{\partial t}+a(t, x) \frac{\partial u}{\partial x}, & u \in H(\beta, Q), \\
u=0, & \partial Q_{-},\end{cases}
\end{aligned}
$$

$u$ represents the concentration of nutrients or of the hormones and $a$ the convective speed. The variational reduction method consists in introducing the following finite dimensional spaces $M\left(\Gamma_{\epsilon}\right)=$ $\operatorname{span}\left(\left\{q_{i}\right\}_{i=0}^{i=m}\right)$ and $M\left(\Gamma_{\eta}\right)=\operatorname{span}\left(\left\{p_{j}\right\}_{j=0}^{j=m^{\prime}}\right)$ and a product space $H\left(\beta, Q_{1}\right) \times M\left(\Gamma_{\epsilon}\right) \otimes_{0}$ $H^{1}(\delta, \theta) \times M\left(\Gamma_{\eta}\right) \otimes_{0} H^{1}(\theta, 1)$ where $\Gamma_{\epsilon}=\left(\frac{1}{2}-\epsilon, \frac{1}{2}+\epsilon\right) \times\{\delta\}, \Gamma_{\eta}=\left(\frac{1}{2}-\eta, \frac{1}{2}+\eta\right) \times\{\theta\}$ and $H^{1}(\delta, \theta)$ is a Sobolev space on $(\delta, \theta)$. We consider the unbounded operator $\frac{d}{d t}: D\left(\frac{d}{d t}\right) \subset L^{2}\left(\Gamma_{\epsilon} \cup\right.$ $\left.\Gamma_{\eta}\right) \rightarrow L^{2}\left(\Gamma_{\epsilon} \cup \Gamma_{\eta}\right)$ such that $d(\varphi, \psi)=\left(\frac{d}{d t} \varphi / \psi\right)_{\Gamma_{\epsilon} \cup \Gamma_{\eta}}$ for all $\psi \in L^{2}\left(\Gamma_{\epsilon} \cup \Gamma_{\eta}\right)$ and $D\left(\frac{d}{d t}\right)$ denotes the domain of definition. We can choose for example $q_{0}(t)=1$ and $q_{j}(t)=\sin \left(j \pi\left(\frac{t-\frac{1}{2}}{\epsilon}+1\right)\right)$ for all $1 \leq j \leq m$ and in the same way $p_{0}(t)=1$ and $p_{j}(t)=\sin \left(j \pi\left(\frac{t-\frac{1}{2}}{\eta}+1\right)\right)$ for all $1 \leq j \leq m^{\prime}$. We prove that the functions $p_{j}$ are orthogonal with respect to both the $L^{2}\left(\Gamma_{\epsilon}\right)$ scalar product and the $L^{2}\left(\Gamma_{\epsilon}, a(\bullet, \delta) d t\right)$ and the functions $q_{j}$ are orthogonal with respect to both the $L^{2}\left(\Gamma_{\eta}\right)$ scalar product and $L^{2}\left(\Gamma_{\eta}, a(\bullet, \theta) d t\right)$. Besides we have $\int_{\frac{1}{2}-\eta}^{\frac{1}{2}+\eta} \frac{\partial}{\partial t}\left(p_{l}(t)\right) p_{i}(t) d t=0 \quad ; \quad 0 \leq i, l \leq m^{\prime}$ and $\int_{\frac{1}{2}-\epsilon}^{\frac{1}{2}+\epsilon} \frac{\partial}{\partial t}\left(q_{k}(t)\right) q_{j}(t) d t=0 \quad ; \quad 0 \leq k, j \leq m$. Then we look for a solution $u=\left(u_{1}, u_{2 M}, u_{3 M}\right)=\left(u_{1}, \sum_{j=0}^{m} q_{j}(t) u_{2 j}(x), \sum_{j=0}^{m^{\prime}} p_{j}(t) u_{3 j}(x)\right)$ satisfying the weak formulation:

- In $Q \backslash\left(Q_{1} \cup Q_{2}\right)$ we look for $u_{3 M} \in M\left(\Gamma_{\eta}\right) \otimes_{0} H^{1}(\theta, 1)$ such as

$$
\begin{array}{r}
\int_{\theta}^{1} \int_{\frac{1}{2}-\eta}^{\frac{1}{2}+\eta} \frac{\partial u_{3 M}}{\partial t} p(t) \varphi(x) d t d x-\int_{\theta}^{1} \int_{\frac{1}{2}-\eta}^{\frac{1}{2}+\eta} u_{3 M} p(t) \frac{\partial a(t, x) \varphi(x)}{\partial x} d t d x \\
-\int_{\frac{1}{2}-\eta}^{\frac{1}{2}+\eta} u_{3 M} p(t) a(t, \theta) \varphi(\theta) d t=\int_{\theta}^{1} \int_{\frac{1}{2}-\eta}^{\frac{1}{2}+\eta} f(t, x) p(t) \varphi(x) d t d x \\
\forall \varphi \in H^{1}(\theta, 1), \varphi(1)=0 . \quad(*)
\end{array}
$$


- In $Q \backslash\left(Q_{1} \cup Q_{3}\right)$ we look for $u_{2 M} \in M\left(\Gamma_{\epsilon}\right) \otimes_{0} H^{1}(\delta, \theta)$ such as

$$
\begin{array}{r}
\int_{\delta}^{\theta} \int_{\frac{1}{2}-\epsilon}^{\frac{1}{2}+\epsilon} \frac{\partial u_{2 M}}{\partial t} q(t) \varphi(x) d t d x-\int_{\delta}^{\theta} \int_{\frac{1}{2}-\epsilon}^{\frac{1}{2}+\epsilon} u_{2 M} q(t) \frac{\partial a(t, x) \varphi(x)}{\partial x} d t d x \\
-\int_{\frac{1}{2}-\epsilon}^{\frac{1}{2}+\epsilon} u_{2 M} q(t) a(t, \delta) \varphi(\delta) d t=\int_{\delta}^{\theta} \int_{\frac{1}{2}-\epsilon}^{\frac{1}{2}+\epsilon} f(t, x) q(t) \varphi(x) d t d x \\
\forall \varphi \in H^{1}(\delta, \theta), \varphi(\theta)=0
\end{array}
$$

The decomposed problem reads: find $\left(u_{1}, u_{2 M}, u_{3 M}\right)$ verifying $(*),(* *)$ and

$$
\begin{cases}\left(\beta / \nabla u_{1}\right)_{2}=f, & \text { in } Q_{1}, \\ b_{1}\left(u_{1}-u_{2 M}, q\right)=\int_{\{x=\delta\}}\left(u_{1}(t, \delta)-u_{2 M}(t, \delta)\right) q(t) a(t, \delta) d t=0, & \forall q \in M\left(\Gamma_{\epsilon}\right), \\ b_{2}\left(u_{2 M}-u_{3 M}, p\right)=\int_{\{x=\theta\}}\left(u_{2 M}(t, \theta)-u_{3 M}(t, \theta)\right) p(t) a(t, \theta) d t=0, & \forall p \in M\left(\Gamma_{\eta}\right) .\end{cases}
$$

The matching conditions on the interface are imposed with a weak formulation. When $a$ is time independent, the decomposed problem becomes

$$
\left\{\begin{array}{l}
a(x) \frac{d\left(u_{30}(x)\right)}{d x}=\frac{1}{2 \eta} \int_{\frac{1}{2}-\eta}^{\frac{1}{2}+\eta} f(t, x) d t d x, \quad p . p \text { on } Q_{3}, \\
u_{30}(\theta)=\frac{1}{2 \eta} \int_{\frac{1}{2}-\eta}^{\frac{1}{2}+\eta} u_{2 M}(t, \theta) d t, \quad \text { on the interface between } Q_{3} \text { and } Q_{2}, \\
a(x) \frac{d\left(u_{20}(x)\right)}{d x}=\frac{1}{2 \epsilon} \int_{\frac{1}{2}-\epsilon}^{\frac{1}{2}+\epsilon} f(t, x) d t d x, \quad p . p \text { on } Q_{2}, \\
u_{20}(\delta)=\frac{1}{2 \epsilon} \int_{\frac{1}{2}-\epsilon}^{\frac{1}{2}+\epsilon} u_{1}(t, \delta) d t, \text { on the interface between } Q_{2} \text { and } Q_{1},
\end{array}\right.
$$

and the classical zero order homogenized problem is obtained when $\varepsilon$ and $\eta$ go to zero. When $a$ is time dependent the decomposed problem becomes

$$
\left\{\begin{array}{l}
{\left[\int_{\frac{1}{2}-\eta}^{\frac{1}{2}+\eta} a(t, x) p_{0}(t) p_{0}(t) d t\right] \frac{d\left(u_{30}(x)\right)}{d x}=\int_{\frac{1}{2}-\eta}^{\frac{1}{2}+\eta} f(t, x) p_{0}(t) d t, \quad \forall \theta \leq x \leq 1,} \\
\int_{\frac{1}{2}-\eta}^{\frac{1}{2}+\eta} a(t, \theta) u_{2 M}(t, \theta) p_{0}(t) d t=u_{30}(\theta) \int_{\frac{1}{2}-\eta}^{\frac{1}{2}+\eta} a(t, \theta) p_{0}(t) d t, \\
\left(\int_{\frac{1}{2}-\epsilon}^{\frac{1}{2}+\epsilon} a(t, x) q_{0}(t) q_{0}(t) d t\right) \frac{d\left(u_{20}(x)\right)}{d x}=\int_{\frac{1}{2}-\epsilon}^{\frac{1}{2}+\epsilon} f(t, x) q_{0}(t) d t, \quad \forall \delta \leq x \leq \theta, \\
\int_{\frac{1}{2}-\epsilon}^{\frac{1}{2}+\epsilon} a(t, \delta) u_{1}(t, \delta) \frac{d\left(u_{20}(x)\right)}{d x} q_{0}(t) d t=u_{20}(\delta) \int_{\frac{1}{2}-\epsilon}^{\frac{1}{2}+\epsilon} a\left(t, \delta \frac{d\left(u_{20}(x)\right)}{d x}\right) q_{0}(t) d t,
\end{array}\right.
$$


and the classical zero order homogenized problem is

$$
\left\{\begin{array}{l}
a\left(\frac{1}{2}, x\right) \frac{d\left(u_{30}(x)\right)}{d x} p_{0}\left(\frac{1}{2}\right)=f\left(\frac{1}{2}, x\right), \quad \forall \theta \leq x \leq 1, \\
u_{20}\left(\frac{1}{2}, \theta\right)=u_{30}(\theta) \\
a\left(\frac{1}{2}, x\right) \frac{d\left(u_{20}(x)\right)}{d x} q_{0}\left(\frac{1}{2}\right)=f\left(\frac{1}{2}, x\right), \quad \forall \delta \leq x \leq \theta \\
u_{1}\left(\frac{1}{2}, \delta\right)=u_{20}(\delta) .
\end{array}\right.
$$

\section{Conclusion and discussion}

To conclude, the 2D initial problem for two branching case has been transformed into a $1 \mathrm{D}$ problem as in [5] for the one branching case. We observe also that the classical zero order approximation corresponds to the weak formulations $(*)$ and $(* *)$ when $\eta$ and $\varepsilon$ equal zero. This justifies the method of asymptotic partial domain decomposition MAPDD applied to study the evolution of the nutrients and hormones concentrations. Our perspectives are to show using numerical simulations that this approach is better than the classical finite differences method to describe this phenomena.

\section{References}

[1] N. Bessonov, V. Volpert. Dynamic models of plant growth. Mathematics and mathematical modeling I. Publibook Paris, (2007).

[2] G.P. Panasenko. Multi-scale modelling for structures and composites. Springer Verlag, (2005).

[3] O. Diallo. Modélisation et simulation numérique de résine réactive dans un milieu poreux. Thèse de doctorat, Université Claude Bernard Lyon 1, (2000).

[4] F. Fontevieille. Décomposition asymptôtique et éléments finis. Thèse de doctorat, Université Claude Bernard Lyon 1, (2004).

[5] M. Picq, J. Pousin. Variational reduction for the transport equation and plants growth. Proccedings of the conference modelling of the heterogeneous materials with applications in constructions and biological engineering, czech technical, University of Prague, (2007). 